\title{
The ratio standard is not adequate for scaling handgrip strength in judo athletes and nonathletes
}

\author{
Wladymir Külkamp', Jonathan Ache-Dias², Rafael Lima Kons ${ }^{3, *}$, Daniele Detanico ${ }^{3}$, Juliano Dal Pupo ${ }^{3}$ \\ ${ }^{1}$ Centre for Health and Sport Sciences, University of Santa Catarina State, Florianópolis, Brazil \\ ${ }^{2}$ Research Group on Technology, Sport and Rehabilitation, Catarinense Federal Institute, Araquari, Brazil \\ ${ }^{3}$ Biomechanics Laboratory, Centre of Sports - CDS, Federal University of Santa Catarina, Florianópolis, Santa Catarina, Brazil
}

This study aimed to analyze if the ratio standard or the allometry is the best scaling methods of handgrip strength (HGS) for body mass (BM) in judo athletes and nonathletes, and to verify if the HGS mean values are higher in judo athletes than nonathletes when the BM effect is removed. One hundred forty-two individuals, being 88 judo athletes and 54 nonathletes participated in this study. The maximal isometric HGS was measured in judo athletes and nonathletes in the preferred hand. Only the allometry method was able to remove the BM effect of HGS. The allometric exponents found for athletes was $b=0.68$ and $b=0.563$ for nonathletes. A common allometric exponent was identified as $(b=0.64)$ for comparing judo athletes and nonathletes. However, neither was able to allow for the comparison between groups due to a distortion of (bias; $P<0.01)$. Thus, an index (HGS INDEX) was proposed to allow within-group (judo athletes and nonathletes) and between-group (judo athletes vs. nonathletes) comparison, removing the effect of BM. In summary, the allometric exponent based on the theory of geometric similarity $(b=0.67)$ was able to remove the BM effect of HGS in judo athletes. An index

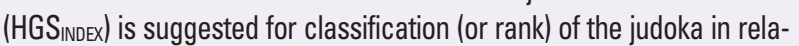
tion to the expected average of HGS.

Keywords: Allometry, Body dimension, Martial arts, Strength

\section{INTRODUCTION}

The handgrip strength (HGS) has frequently been used as a parameter to determine physical and functional performance in the upper limbs. It is known the importance of HGS in activities of daily living to maintain basic and specific needs (Garber et al., 2011), in the area of clinical diagnostics (Karavelioglu et al., 2017; Pua, 2006; Vanderburgh et al., 1995), occupational health environment (Rostamzadeh et al., 2020), and finally applied to the sport context (Ache Dias et al., 2012; Gerodimos et al., 2013; Kons et al., 2018a; Kons et al., 2018b). The HGS plays a decisive role for performance in grappling combat sports such as judo, Brazilian jiu-jitsu, wrestling etc. that require high levels of strength or muscular endurance of the forearm and finger flexor muscles (Andreato et al., 2017; Calmet et al., 2010; Franchini et al., 2013; Gerodimos et al., 2013). In particular, most part of the judo match- es demands for high level of HGS, especially isometric strength of the forearm flexors muscles (Kons et al., 2018b). Isometric HGS has been correlated with effective combat time obtained in official matches in judo athletes (Kons et al., 2018a), indicating that high levels of HGS may allow for the control of the opponent during combat, better maintenance of grip and subsequently the execution of throwing techniques (Calmet et al., 2010).

An important parameter that should be considered in the HGS assessment is the influence of body mass (BM), mainly when using absolute values in comparative approaches. In this sense, the HGS has been commonly scaled for BM using the ratio standard (i.e., HGS/BM) (Branco et al., 2018; Franchini et al., 2018; Franchini et al., 2020). However, this scaling method assumes a direct or linear relationship between HGS and BM (Tanner, 1949), i.e., to admit that any variation of HGS is due exclusively to BM variation (Jaric, 2002; Marković and Sekulić, 2006; Nevill et al., 2009).
${ }^{\star}$ Corresponding author: Rafael Lima Kons (D) https://orcid.org/0000-0003-1615-5464 Federal University of Santa Catarina, Centre of Sports, Biomechanics Laboratory, Florianópolis, Santa Catarina 88040-900, Brazil E-mail: rafakons0310@gmail.com

Received: February 21, 2020 / Accepted: March 24, 2020
This is an Open Access article distributed under the terms of the Creative Commons Attribution Non-Commercial License (https://creativecommons.org/licenses/by-nc/4.0/) which permits unrestricted non-commercial use, distribution, and reproduction in any medium, provided the original work is properly cited. 
This implies in penalizing heavyweight individuals when their performance is compared to lightweight individuals. For this reason, the allometry approach has been suggested as the most appropriate option to allow for the comparison of individuals or groups with different BM. This method assumes that the relationship between biological variables (e.g., HGS) and anthropometric variables (e.g., BM) is nonlinear (Winter, 1992) and thereby using a power function to remove the BM effect according to the exponent of this function. A common theoretical allometric exponent $(b=0.67)$ has been supported by some researchers, based on the theory of geometric similarity (McMahon, 1984), but other exponents have also been proposed (Jaric, 2002). For example, Vanderburgh et al. (1995) proposed an allometric exponent of 0.51 for the comparison of HGS of healthy individuals.

The HGS, as previously mentioned, is an important tool used for the evaluation of athletes and nonathletes. However, an important aspect to be considered is that muscle strength is an adaptable (trainable) physical capacity (i.e., increases as a result of training); thus, it is reasonable to assume that higher HGS values should be expected in judo athletes than nonathletes. This implies, assuming this hypothesis, that the relationship between HGS and BM may be different between these two populations and consequently leading to different allometric exponents. Therefore, specific exponents seem to be necessary for each specific group, limiting the use of values proposed by Vanderburgh et al. (1995) for comparison of HGS in athletes. A previous study showed that peak HGS did not differ between judo athletes and nonathletes when considered mean absolute values (Ache Dias et al., 2012); however, from the allometric point of view this type of comparison still needs to be investigated.

Therefore, the present study aimed to analyze what is the most appropriate scaling method (ratio standard or allometry) of HGS for BM in judo athletes and nonathletes, hence, to verify whether it is possible to compare the HGS between judo athletes and nonathletes when BM effect is removed.

\section{MATERIALS AND METHODS}

\section{Study approach}

Two hypotheses originated from the objectives of this current study. The first is that the ratio standard method does not adequately remove the effect of BM on HGS in judo athletes and nonathletes, and the allometric method would be a suitable alternative for a performance comparison of individuals with different $\mathrm{BM}$. The second hypothesis is that a different relationship be- tween BM and HGS should be expected when evaluating judo athletes and nonathletes, because muscle strength is a trainable capacity, i.e., a higher HGS per BM unit should be expected in well-trained individuals. Different exponents should be observed for judo athletes and nonathletes by the allometric method, then, to compare the HGS between the groups should be not possible.

A cross-sectional study with two approaches was developed to test the hypotheses. In the first approach, the values obtained by ratio standard and allometric methods were compared, whereas in the second approach a new index for HGS was developed aiming to compare athletes and nonathletes of different BM from a common metric.

\section{Participants}

Eighty-eight judo athletes (height, $175.03 \pm 8.58 \mathrm{~cm}$; BM, $72.83 \pm 13.87$ [50-100] kg; age, $22.42 \pm 6.54$ years) and 54 nonathletes (height, $175.86 \pm 7.51 \mathrm{~cm}$; BM, $74.38 \pm 11.62$ [50-100] $\mathrm{kg}$; age, $22.94 \pm 5.45$ years) participated in this study. The judo athletes (time of practice of $10.1 \pm 4.1$ years) attended training sessions on at least 5 days a week and had a minimum of 6 years of experience in their sport and were competing at a state or national level. The group of nonathletes was composed of healthy university students that did not practice any martial art or sport modality regularly. None of the participants reported any upper limb muscular-skeletal injury that occurred in the 6 months prior to the study. All participants received a detailed verbal explanation of the purpose, methods and potential risks/benefits of this study and signed a written informed consent form agreeing to participate. This study was approved by the Research Ethics Committee of local university (number: 1.957.840), according to Declaration of Helsinki.

\section{Procedures}

Initially, BM was assessed using a digital scale $(0.1 \mathrm{~kg}$ of accuracy). The measure was performed before the HGS evaluation by an experienced evaluator (level 1 of the International Society for Advancement in Kinanthropometry).

The maximal isometric HGS test followed the procedures adopted by the American Society of Hand Therapy (ASHT). Participants were seated with their spines erect, maintaining a knee flexion angle of $90^{\circ}$. The shoulder was positioned in adduction and neutral rotation, and the elbow was flexed at $90^{\circ}$, with the forearm in half-pronation and with a neutral grip. Judo athletes and nonathletes were instructed to hold the handgrip dynamometer adjusted in the second handle position (Saehan 134, SH5001 model, 
Saehan Corp., Masan, Korea) and perform three attempts with maximum effort for $3 \mathrm{sec}$ in the preferred hand, with a rest period of $30 \mathrm{sec}$ between each trial. We used the higher value from the three trials for further analysis. We assessed the reliability of the HGS test using the three trials, and the intraclass correlation coefficient was 0.93 for the preferred.

\section{Data analyses and statistics}

\section{Ratio scaling and allometric modeling}

The relationship between HGS (independent variable) and BM (dependent variable) of judo athletes and nonathletes was initially analyzed from a linear regression established between the variables. The HGS normalized from the ratio standard method was obtained by dividing the HGS of the individuals by their BM (equation 1).

$$
\mathrm{HGS}_{\mathrm{RS}}=\mathrm{HGS} * \frac{1}{\mathrm{BM}} \text { (equation 1) }
$$

where, HGS, handgrip strength; BM, body mass; RS, ratio standard.

The allometric modeling of variables was performed using the power functions (equation 2) and their linearization, based on linear and logarithmic (or log-linear) regressions (equation 3) established from the calculation of natural logarithms (ln) of BM and HGS, using the $\operatorname{lnBM}$ and $\ln H G S$ as independent and dependent variables, respectively.

$$
\begin{gathered}
\mathrm{HGS}=\mathrm{A} * \mathrm{BM}^{\mathrm{b}} * \mathrm{E} \text { (equation 2) } \\
\ln \mathrm{HGS}=\ln \mathrm{BM} * \mathrm{~b} *+\ln \mathrm{E} \text { (equation } 3 \text { ) }
\end{gathered}
$$

where, ln, natural logarithm; HGS, handgrip strength; BM, body mass; "a" and "b" are regression coefficients; A, ea, e, Euler' constant; E, residuals.

The allometric adjusted HGS ( $\left.\mathrm{HGS}_{\mathrm{AL}}\right)$ of each participant was obtained according to equation 4 .

$$
\left.\mathrm{HGS}_{\mathrm{AL}}(\mathrm{kg})=\frac{\mathrm{HGS}}{\mathrm{BM}^{\mathrm{b}}} \text { (equation } 4\right)
$$

\section{Usefulness of ratio standard and allometric models}

The main criterion for verifying the usefulness of ratio standard and allometric models to remove the BM effect for both groups was the significance of the correlation between the adjusted HGS ( $\mathrm{HGS}_{\mathrm{RS}}$ and $\mathrm{HGS}_{\mathrm{AL}}$ ) and BM. No significant correlation indicates the adequacy of the method. If a successful model was confirmed in the first criterion, additional regression diagnostic criteria were used considering normality, homoscedasticity (homogeneous variance) and the absence of self-correlation in the residuals (Batterham and George, 1997; Cleather, 2006; Pua, 2006; Vanderburgh and Dooman, 2000; Zoeller et al., 2008). The normality of residuals distribution was tested by Shapiro-Wilk test $(P>0.05)$. Homoscedasticity of the residuals was verified by visual inspection and Pesarán-Pesarán test (absence of correlation between standardized residues and estimated values; $P>0.05$ ). Self-correlation in the residuals was tested by Durbin-Watson statistic, where the value of the test (dw) must be close to 2 to guarantee absence of self-correlation (critical values between the "dU" and "4-dU").

\section{Comparison of HGS between groups}

The HGS absolute values of judo athletes and nonathletes were initially compared using an independent $t$-test for comparison of means. Afterward, the slope coefficients of linear regression (BM vs. HGS) were compared to verify if the correlation between HGS and BM was the same between groups (athletes and nonathletes). Different values of slope would indicate different relationships between BM and HGS in each group.

From the allometric point of view, in case of difference between the exponent of the groups, the comparison of HGS between groups would be performed from a multiple log-linear regression model, adding two covariates to the traditional log-linear model, one of them related to the group and the other a covariate of interaction (Winter, 1992). From the significance ( $P$-value) of each term of multiple log-linear regressions, it would be possible to define a

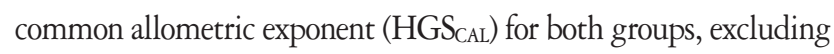
an interaction term if nonsignificant $(P>0.05)$. The difference between groups was expressed by the different power function constant multiplier (A - equation 2). For judo athlete's "A" was calculated from the antilogarithm of the constant of common log-linear equation ('a'-, equation 5), after excluding an interaction factor. For nonathletes "A" was obtained by the addition of the constant of the common log-linear equation to the value of the coefficient related to group $(a+c$, equation 5$)$, with the subsequent calculation of the antilogarithm of the result of this addition.

$\ln H G S=\ln a+(b \times \ln B M)+(c \times$ group code $)+(d x$ interaction $)+\ln E$ (equation 5$)$

where, group code: "1" for judo athletes, "2" for nonathletes; interaction, $\ln B M x$ group code.

\section{Proposal of a common index (HGS INDEX)}

A common index for classification of HGS values in judo ath-

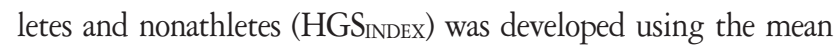


$\mathrm{HGS}_{\mathrm{AL}}$ of each group. This index represents the ratio between the individual HGS value of the individual in relation to the expected mean for his group (judo athletes or nonathletes). To make the index more explanatory, the ratio was decreased from 1 and multiplied by 100 , thereby any value above, below or equal to zero represents its proximity in percentage units to the expected mean (equation 6).

$$
\operatorname{HGS}_{\mathrm{INDEX}}(\%)=\left(\frac{\text { individual } \mathrm{HGS}_{\mathrm{AL}}}{\text { mean of group } \mathrm{HGS}_{\mathrm{AL}}}-1\right) \times 100 \text { (equation 6) }
$$

Considering that the mean of each group is used in each respective equation, comparisons can be performed between individuals of the same group (within-group) or between different groups (between-group), even though the allometric exponents of each group are not the same. For example, a value of $\mathrm{HGS}_{\text {INDEX }}=5$ means that the individual would be $5 \%$ above the expected average when the BM effect is removed, regardless of which group he is part of (judo athlete or nonathlete). Additionally, a four-level classification scale

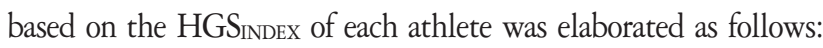
HGS $_{\text {INDEX }} \geq 15$ (superior); $0<$ HGS $_{\text {INDEX }}<15$ (median-superior); $0>\mathrm{HGS}_{\text {INDEX }}>-15$ (median-inferior); and $\mathrm{HGS}_{\text {INDEX }} \leq-15$ (inferior). In this classification model, the zero of the scale was interpreted as referring to performance identical to the expected average for each specific BM range. Thus, performance levels can be used for an overall ranking, while the HGS INDEX can be useful for individual comparisons within each of the four levels of performance.

All statistical analysis was performed in IBM SPSS Statistics ver. 23.0 (IBM Co., Armonk, NY, USA) with a significance level set at 5\%. Post hoc power analysis was performed using the $\mathrm{G}^{*}$ power 3.1 software, achieving values greater than 0.97 . Effect size was presented as Cohen $r$, where 0.1 means small effect, 0.3 means medium effect and 0.5 means large effect (Cohen, 1988). Pearson correlation coefficient " $r$ " was interpreted as effect size in correlations analysis between variables. A method of conversion of a $t$-value into an $r$-value was used as effect size when comparing means. Thus, statistical power was calculated considering sample size, effect size, $t$-values and degrees of freedom, according to the statistical test, always assuming the given alpha (0.05).

\section{RESULTS}

Absolute values of HGS did not differ between judo athletes $(54.38 \pm 12.02 \mathrm{kgf})$ and nonathletes $(54.08 \pm 10.44 \mathrm{kgf})(P>0.05$, $\mathrm{ES}=0.012)$. The relationship between HGS and BM was confirmed for judokas $(P<0.05, \mathrm{ES}=0.58)$ and nonathletes $(P>0.05$, ES= 0.51 ) when assuming raw values (Fig. $1 \mathrm{~A}$ ) or logarithmic values
$(P<0.05, \mathrm{ES}=0.57$ for judokas and $P<0.05, \mathrm{ES}=0.48$ for nonathletes) (Fig. 1B).

Slope coefficients of regression lines of BM and raw HGS (Fig. 1A) were not statistically different $(P>0.05, \mathrm{ES}=0.0003)$ between judo athletes $(b=0.68)$ and nonathletes $(b=0.563)$, suggesting a similar linear relationship between these variables in each group. Although the difference between allometric exponents was not significant, $(P>0.05, \mathrm{ES}=0.04)$, the comparisons between-group were not possible due to the different averaged values between groups (3.09 \pm 0.56 for judokas, $4.79 \pm 0.81$ for nonathletes, $P<$ $0.05, \mathrm{ES}=0.78$ ).

The HGS was scaled for BM considering the ratio standard and the allometric adjustment. The ratio standard (Fig. 1C) did not remove the BM effect on HGS in both groups, considering the significant correlation between $\mathrm{BM}$ and $\mathrm{HGS}$ scaled using ratio standard, for judokas $(P<0.05 ; \mathrm{ES}=0.31)$ and non-judokas $(P<$ 0.05; ES $=0.37)$. On the other hand, no correlation was observed between BM and HGS allometrically adjusted $(P>0.05)$, indicating that the allometric method was able to adequately remove the BM effect, for both groups (Fig. 1D).

The adequacy of allometric adjustment was confirmed based on regression diagnosis criteria, presenting normality $(P>0.05)$, homoscedasticity $(P>0.05)$ and the absence of self-correlation ( $\mathrm{dw}$ $\sim 2$ ) in the residual distribution for both judo athletes and nonathletes (Fig. 1D). The allometric exponent found for judo athletes in the present study $(b=0.68)$ was very close to the theoretical value $(b=0.67)$ proposed by the geometric similarity theory (13); thus, the 0.67 exponent was adopted for additional analyses.

Considering that the observed allometric exponents for judokas and nonathletes were different (Fig. 1B), i.e., HGS $_{\mathrm{AL}}$ of the groups could not be directly compared (Fig. 1D), the comparison between groups was performed using a multiple log-linear model. It was observed that both the term of equation related to group and interaction term did not present statistical significance $(P>0.05)$. Thus, the two nonsignificant terms were removed from the equation and a common allometric exponent (HGS CAL $_{\text {) was identified }}$ through the backward method $(b=0.641)$. With regard to the comparison between the groups, the allometric adjustment does not allow for the identity of the difference in HGS between judo athletes and nonathletes. This was made clearer when the interaction term of the equation was removed, in which the observed value for the multiplicative factor of the power function ("A") was 3.43 for judo athletes, practically the same value observed for nonathletes $(b=3.38)$. Although the common allometric exponent (HGS CaL, 0.641 ) could be used to compare judokas and nonath- 


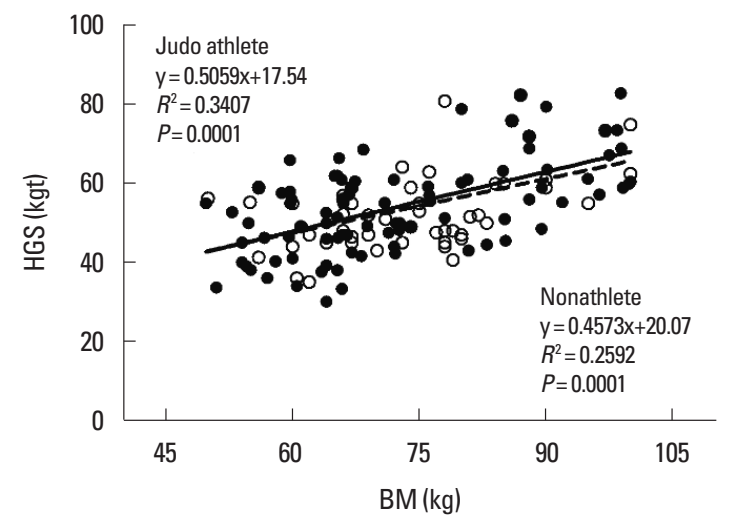

A
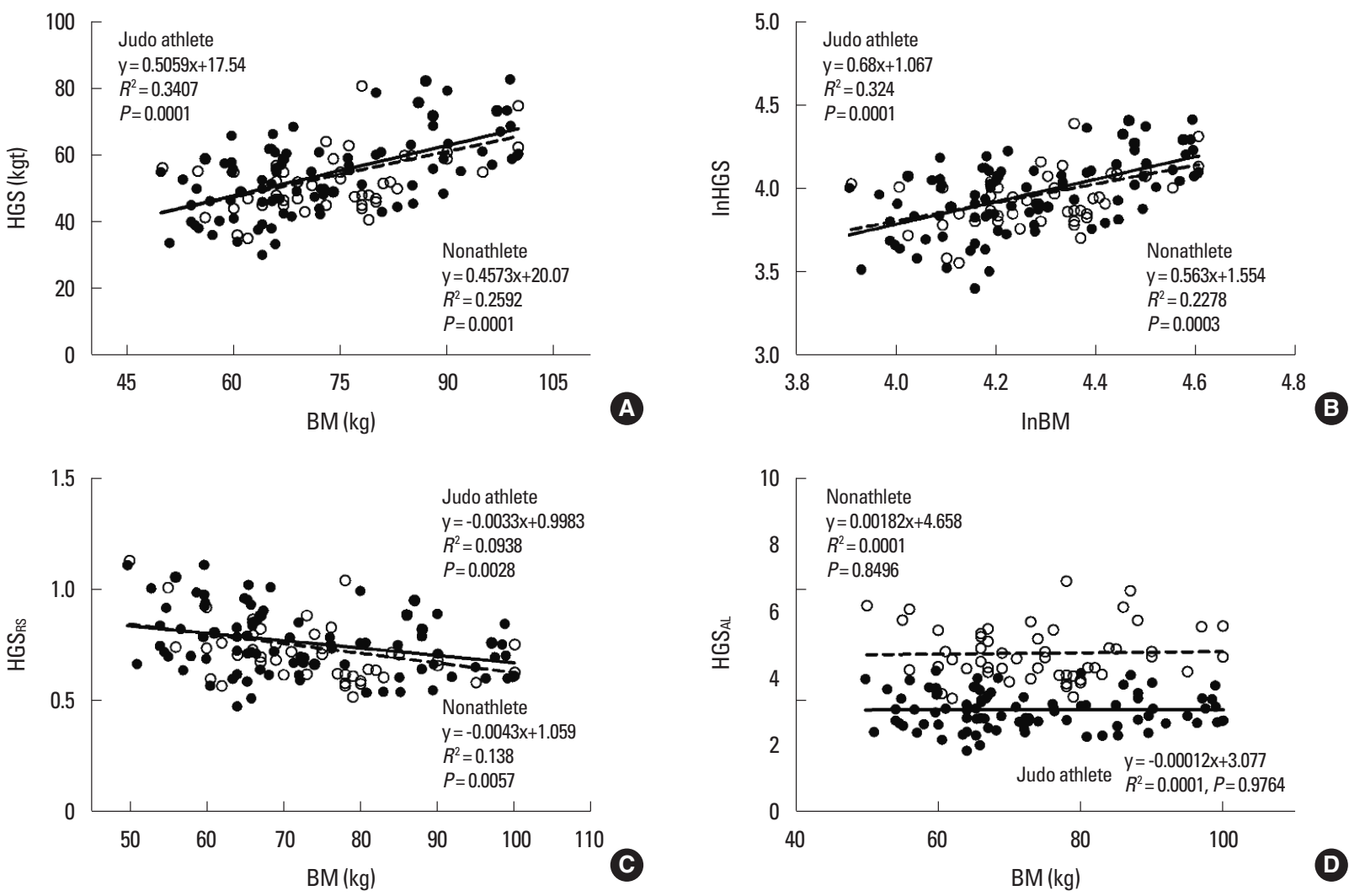

Fig. 1. (A) Regression between body mass (BM) and absolute handgrip strength (HGS) for judo athletes and nonathletes. (B) Regression between In of BM (InBM) and In of HGS (InHGS) for judo athletes and nonathletes. (C) Correlation between BM and ratio standard of handgrip strength (HGS RS) for judo athletes and nonathletes. (D) Correlation between BM and allometric adjusted HGS (HGSAL). In, natural logarithm. Filled circle indicates athletes and open circles indicate nonathletes.

letes, additional analysis showed that its use would imply significant and proportional distortion (bias) when comparing HGS INDEX of both groups. As observed in Fig. 2, the distance of individual performance in relation to the average of the groups is different when using group-specific exponents (HGS $\mathrm{AL}_{\mathrm{L}}$ ) compared to the

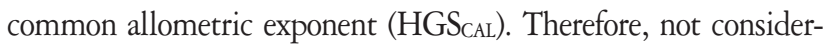
ing this difference would penalize heavier individuals and give advantage for lighter ones when comparing judokas and, vice-versa when comparing nonathletes.

Thus, a common index for within-group and between-group comparison was established, considering the $\mathrm{HGS}_{\mathrm{AL}}$ averages of each group (equations 7 and 8). Fig. 3 shows how this index allows for a comparison, even between individuals from groups with different allometric exponents. Clearly, this is only possible because the allometric adjustment guarantees a constant average over the entire $\mathrm{BM}$ range (slope $=0, P>0.05$ ).

$$
\text { HGS }_{\text {INDEX_athletes }}=\left(\frac{\left(\frac{\mathrm{HGS}}{\mathrm{BM} 0.67}\right)}{3.086}-1\right) * 100 \text { (equation 7) }
$$

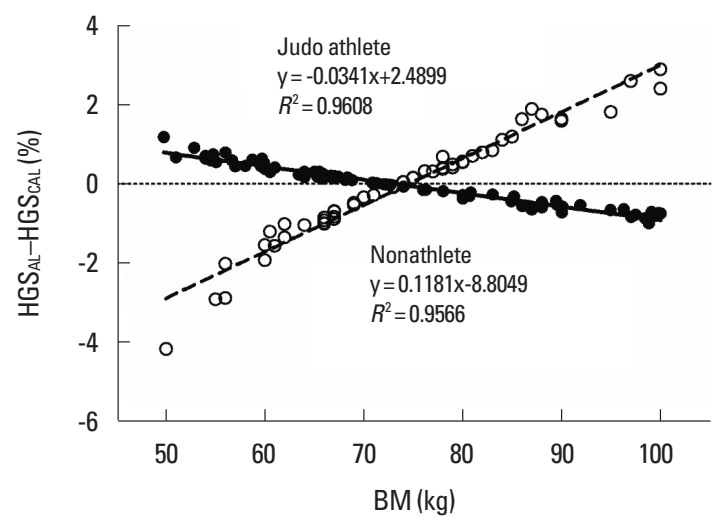

Fig. 2. Correlation between body mass and the difference of the performance classification provided by allometric handgrip strength ( $\mathrm{HGS}_{\mathrm{AL}}$ ) and common allometric exponent (HGS CAL) for judo athletes (filled circle) and nonathletes (open circle).

$$
\mathrm{HGS}_{\text {INDEX_non-athletes }}=\left(\frac{\left(\frac{\mathrm{HGS}}{\left.\mathrm{BM}^{0.563}\right)}\right.}{4.794}-1\right) * 100 \text { (equation 8) }
$$

where, numerator, HGS allometric; denominator, mean of group allometric HGS. 


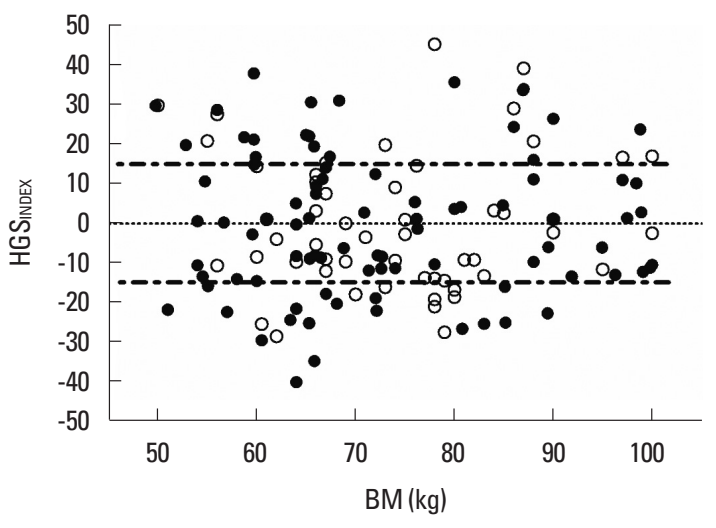

Fig. 3. Common HGS index (HGS InDEx) for comparison of HGS between judo athletes (filled circle) and nonathletes (open circle), removing the body mass effect and the difference between allometric exponents ("b"). HGS, handgrip strength.

A four-level rating scale was proposed based on $\mathrm{HGS}_{\text {INDEX }}$ standard deviation (SD) of judo athletes $(\mathrm{SD}=18 \%)$ and nonathletes $(\mathrm{SD}=17 \%)$, since it was not possible to visually determine representative thresholds. A common value of 15\% ( 1 SD) was used to determine the thresholds between lower and upper limits, and the ZERO of math scale coincided with a performance equal to the expected mean for each group (Fig. 3).

\section{DISCUSSION}

The first objective of this study was to analyse which is the best method, ratio standard or allometry, for scaling the HGS for BM in judo athletes and nonathletes. According to our results, only allometric scaling was able to remove the BM effect of HGS for both, athletes and nonathletes, confirming the first hypothesis. In addition, even when using a common allometric exponent, it was not possible to compare the HGS between these groups, confirming the second hypothesis. Therefore, it is not possible to state that judo athletes are stronger or weaker than nonathletes, even when BM effect was removed. These results allow us to develop a common index for within-group and between-group comparison.

Allometry has been commonly used in physical assessment, but it is still neglected when HGS is analyzed. Some studies have investigated the use of allometry in HGS data (Karavelioglu et al., 2017; Pua, 2006; Vanderburgh et al., 1995), especially in athletes, but many others studies have been using the ratio standard (Branco et al., 2018; Franchini et al., 2018; Franchini et al., 2020). Vanderburgh et al. (1995) was one of the first to investigate the use of allometry in HGS data and found an allometric exponent of 0.54 for male university students, a value very close to what was found in the present study $(b=0.56)$. The allometric exponent found for judo athletes in the present study $(b=0.68)$ is very close to the exponent based on the theory of geometric similarity (McMahon, 1984) observed in other experiments (Jaric, 2002).

Recently, a classificatory table for adult judo athletes was proposed according to the HGS magnitude in absolute and relative terms (ratio standard) (Franchini et al., 2018); however, our data suggest that ratio standard is not the best scaling method because it does not remove adequately the BM effect. In this sense, the normative values should be analyzed with caution when comparing judo athletes with different $\mathrm{BM}$ categories.

Trying to verify if judo athletes are stronger than nonathletes, an allometric model of common exponent was proposed resulting in one allometric exponent (HGS CAL $\left._{1} b=0.64\right)$. Although the common allometric exponent could be used to compare individuals from both groups, its use would imply significant distortion (bias), when compared to expected average $\mathrm{HGS}_{\mathrm{AL}}$ (Fig. 3). Allometry penalizes heavier and lighter athletes and vice-versa. This may occur because the strength is a trainable capacity and the relationship between BM and HGS should be different between trained and nontrained individuals. Therefore, it is not possible to state if judo athletes are stronger than nonathletes. In this sense, our results support the findings of Ache Dias et al. (2012), in which judo athletes presented higher strength endurance in the hands (grip action) than nonathletes. These results may be expected since judo athletes generally need to use strength endurance in the hands to maintain the control of opponent throughout the combat.

Some limitations of study were important to highlight. First,

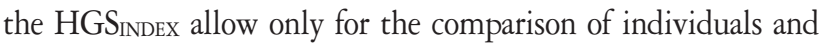
not comparison of groups, since the approach is based on the residuals whose means is zero. Second, the HGS evaluation follows the recommendations of ASHT; thus, the values of HGS, the allometric exponents and the HGS INDEX are useful only when the same protocol of HGS evaluation was followed.

In conclusion, the allometry was able to remove the BM effect of HGS of judo athletes and the allometric exponent obtained in this study for judokas (0.68) support the hypothesis based on the theory of geometric similarity. The BM effect was also adequately removed for nonathletes by allometric scaling, even though a different allometric exponent had been observed (0.56). Although from a practical point of view the difference seems not to be significant, it is not possible to state if and how much judokas are stronger or weaker than nonathletes, even when allometric adjustment is used. The proposed HGS INDEX allowed within-group (judo athletes and nonathletes) and between-group (judo athletes vs. 
nonathletes) comparison, removing the effect of BM.

We recommend the use of allometric scaling with exponent of 0.67 to compare the HGS of judokas, regardless of the athlete's weight category, as well as exponent of 0.56 to compare the HGS of nonathletes. Establishing an HGS normative values is not an easy task due to its relationship with BM, then, we encourage to

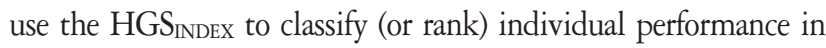
four categories (superior, mean-superior, mean-inferior, and inferior), according to the equations presented for judo athletes and nonathletes.

\section{CONFLICT OF INTEREST}

No potential conflict of interest relevant to this article was reported.

\section{REFERENCES}

Ache Dias J, Wentz M, Külkamp W, Mattos D, Goethel M, Borges Junior $\mathrm{N}$. Is the handgrip strength performance better in judokas than in non-judokas? Sci Sports 2012;27:9-14.

Andreato LV, Lara FJD, Andrade A, Branco BHM. Physical and physiological profiles of brazilian jiu-jitsu athletes: a systematic review. Sports Med Open 2017;3:9.

Batterham AM, George KP. Allometric modeling does not determine a dimensionless power function ratio for maximal muscular function. J Appl Physiol (1985) 1997;83:2158-2166.

Branco BH, Andreato LV, Ribeiro ED, Oliveira HG, Almeida FN, Nardo Junior N. Development of tables for classifying judo athletes according to maximal isometric strength and muscular power, and comparisons between athletes at different competitive levels. Sport Sci Health 2018;14:607-614.

Calmet M, Miarka B, Franchini E. Modeling of grasps in judo contests. Int J Perform Anal Sports 2010;10:229-240.

Cleather DJ. Adjusting powerlifting performances for differences in body mass. J Strength Cond Res 2006;20:412-421.

Cohen J. Statistical power analysis for the behavioural sciences. 2nd ed. New York (NY): Academic Press; 1988.

Franchini E, Artioli GG, Brito CJ. Judo combat: time-motion analysis and physiology. Int J Perform Anal Sport 2013;13:624-641.

Franchini E, Schwartz J, Takito MY. Maximal isometric handgrip strength: comparison between weight categories and classificatory table for adult judo athletes. J Exerc Rehabil 2018;14:968-973.

Franchini E, Schwartz J, Takito MY. Maximal isometric handgrip strength in judo athletes from different age groups. Sport Sci Health 2020;16:
93-98.

Garber CE, Blissmer B, Deschenes MR, Franklin BA, Lamonte MJ, Lee IM, Nieman DC, Swain DP; American College of Sports Medicine. American College of Sports Medicine position stand. Quantity and quality of exercise for developing and maintaining cardiorespiratory, musculoskeletal, and neuromotor fitness in apparently healthy adults: guidance for prescribing exercise. Med Sci Sports Exerc 2011;43:13341359.

Gerodimos V, Karatrantou K, Dipla K, Zafeiridis A, Tsiakaras N, Sotiriadis $S$. Age-related differences in peak handgrip strength between wrestlers and nonathletes during the developmental years. J Strength Cond Res 2013;27:616-623.

Jaric S. Muscle strength testing: use of normalisation for body size. Sports Med 2002;32:615-631.

Karavelioglu MB, Harmanci H, Caliskan G. Gender differences in hand grip strength of the child athletes by using absolute, ratio and allometric scaling methods. Biomed Res 2017;28:1533-1537.

Kons RL, Dal Pupo J, Ache-Dias J, Detanico D. Female judo athletes' physical test performances are unrelated to technical-tactical competition skills. Percept Mot Skills 2018a;125:802-816.

Kons RL, Pupo JD, Ache-Dias J, Garcia T, da Silva RR, Katicips LFG, Detanico D. Effect of official judo matches on handgrip strength and perceptual responses. J Exerc Rehabil 2018b;14:93-99.

Marković G, Sekulić D. Modeling the influence of body size on weightlifting and powerlifting performance. Coll Antropol 2006;30:607-613.

McMahon TA. Muscles, reflexes, and locomotion. Princeton (NJ): Princeton University Press; 1984.

Nevill A, Tsiotra G, Tsimeas P, Koutedakis Y. Allometric associations between body size, shape, and physical performance of Greek children. Pediatr Exerc Sci 2009;21:220-232.

Pua YH. Allometric analysis of physical performance measures in older adults. Phys Ther 2006;86:1263-1270.

Rostamzadeh S, Saremi M, Fereshteh T. Maximum handgrip strength as a function of type of work and hand-forearm dimensions. Work 2020; 65:679-687.

Tanner JM. Fallacy of per-weight and per-surface area standards, and their relation to spurious correlation. J Appl Physiol 1949;2:1-15.

Vanderburgh PM, Dooman C. Considering body mass differences, who are the world's strongest women? Med Sci Sports Exerc 2000;32:197201.

Vanderburgh PM, Mahar MT, Chou CH. Allometric scaling of grip strength by body mass in college-age men and women. Res Q Exerc Sport 1995; 66:80-84.

Winter EM. Scaling: partitioning out differences in size. Pediatr Exerc Sci 1992;4:296-301. 
Zoeller RF, Ryan ED, Gordish-Dressman H, Price TB, Seip RL, Angelopoulos TJ, Moyna NM, Gordon PM, Thompson PD, Hoffman EP. Allome- tric scaling of isometric biceps strength in adult females and the effect of body mass index. Eur J Appl Physiol 2008;104:701-710. 